\title{
Modelling rail track performance: an integrated approach
}

\section{Y.-J. Zhang, M. Murray and L. Ferreira}

\section{Polack, member}

Further to their original paper, can the authors answer the following questions.

(1) Can the authors provide more detail on how the ITDM model can be used to model a line of route? How is the route broken down into sections for analysis? Must track geometry (radius, cant, cant deficiency, etc.) be constant for each section?

(2) How sensitive to speed is sleeper failure and substructure failure? In the UK when the network operator imposes a speed restriction due to 'condition of track' it frequently imposes a lower speed for freight traffic than for passenger. Does the ITDM model demonstrate that such discrimination is justified?

(3) Paragraph 29 states that sleeper damage is more strongly dependent on the number of axle passes than the axle weight. What effect, if any, does train length and axle spacing have on sleeper damage?

(4) Does the ITDM model allow for the effect of high-impact forces from wheel flats or overloaded wagons?

(5) Rail wear and rail breaks are topical subjects in the UK. Is the model able to predict rail breaks? How sensitive is sleeper damage and track roughness to different rail sections? What assumptions have been made in the model concerning rail/wheel profile and rail/wheel interface?

(6) Has any attempt been made to develop the model as a basis for charging a freight train operator for use of a route?

Author's reply

(1) The model was developed for analyses of specific segments. To perform route analysis, the track will have to be firstly segmented into 'homogeneous' track segments, then each segment can be analysed using the model. Alternatively, track segments with similar features can be grouped together to do the analysis. The track segmentation and grouping functions were not included in the model.

(2) Sensitivity of sleeper failure to speed is dependent on track condition (mainly track geometry condition), especially for concrete sleepers. If the track condition is poor, the dynamic loading caused by speed may lead to the cracking limit of concrete sleepers being exceeded and lead to consequent damage to sleepers.

To answer the second part of the question, traffic types (unit train traffic, mix freight traffic, passenger dominant traffic, etc.) are inputs to the model. Different traffic types cause different track degradation rates due to variation in loading conditions. Passenger dominant traffic is usually associated with lighter axle loads than freight traffic. When the track condition is poor, such as might lead to imposition of a 'slow order', freight trains will do more damage to the track than passenger trains if they travel at the same speed; the model accounts for this effect. However, the risk of derailment is often more important than track damage in determining a 'slow order'. A freight train with greater variation in loading is more likely to derail than a passenger train if the track is misaligned or has too much unbalanced superelevation, or cant, on curves. Of course, loading condition is not the only reason why freight trains are discriminated in this way. Many other factors are also influential, for example bogie structures, the type of suspension and its characteristics.

(3) There is a misinterpretation here. The furst part of paragraph 29 states that this is the case for the specific case studied. However, the second half of the paragraph explains that the age of timber sleepers must also be considered here. So when timber sleepers are new, heavier axle loads with fewer passes (e.g. $30 \mathrm{t} \times 100000$ passes $=3 \mathrm{mgt}$ ) will not necessarily do more damage to the sleepers than lighter axles with more passes $(20 t \times 150000$ passes $=3 \mathrm{mgt}$ ), assuming that the sleeper's safe working stresses are not exceeded by the higher axle load. However, when the sleepers are old (say 15 years old and moderately decayed), the first traffic scenario will do much more damage to the sleepers.

(4) The effect was collectively included in the model. The distribution of dynamic forces applied by the axles and the change in the distribution over time were built into the model. However, the effect was not explicitly specified for each individual source that causes the high-impact forces. The model could be improved in this area.

(5) The model does not predict rail breaks. To the best of the 
authors' knowledge, no models exist which can predict when rail breaks will occur, although there are models which can predict the occurrence of defects in rails, which may lead to breaks.

Different rail sections will have different bending stiffnesses (vertical and lateral). This will affect the calculated stress in sleepers and result in different sleeper degradation rates. However, at the time of writing the paper, the specific sensitivity of sleeper damage to rail section size was not studied, although the model has the capability to do it. Track roughness is not sensitive to rail section size, because within a section of track being studied in the model the rails are normally a uniform size.

Nevertheless, the model has the capability to examine the effect of variation in rail size.
No assumptions have been made in the model concerning rail/wheel profile and rail/wheel interface. The model mainly concerns track characteristics, not those of the vehicles. Rail/wheel interaction itself is a challenging subject which has warranted many studies.

(6) The model was not developed for that purpose. However, the user may reference the model results for charging freight traffic or passenger traffic. An additional model developed at Queensland University of Technology has incorporated ITDM into a system enabling forecasting of maintenance needs and associated costs; this process can lead to determination of charges. Please note, however, that a comprehensive track charging system should also consider loading patterns and cargo types. 